\title{
A 6.6 GHz Monotron with a Coaxial Bragg Reflector
}

\author{
Joaquim J. Barroso and Joaquim P. Leite Neto \\ Associated Plasma Laboratory, National Institute for Space Research - INPE \\ 12201-970 São José dos Campos, SP, Brazil
}

\begin{abstract}
A coaxial line periodically loaded by circular disks on the inner conductor is specifically designed to act as Bragg reflector to prevent $r$-f fields from leaking out of a $6.6 \mathrm{GHz}$ monotron Design criteria to ensure a stopband as wide as possible are presented and a discussion is given on how band gap width, center frequency, and reflectivity arise from the geometry of the periodic structure.
\end{abstract}

Keywords: periodic waveguide, disk-loaded coaxial line, Bragg reflector, microwave generation, monotron

\section{Introduction}

Bragg reflectors are highly reflective mirrors consisting of repeated periodic structures, either in the form of a periodically loaded metallic waveguide, a multilayer film or an arrayed dielectric. These structures exhibit so-called stop band phenomena leading to frequency-selective reflection, with applications in Gunn oscillators for integrated circuits [1], tunable lasers [2], and high-power microwave masers [3]. A stop band appears at a chosen frequency when the periodic length of the structure, i. e. the spacing between discontinuities, is one-half wavelength in the unloaded guide or in the dielectric without grating.

In this report we show the design of a coaxial Bragg reflector for application in a monotron, the simplest of the microwave tubes [4]. It consists (Fig.1) of a TM-mode cavity driven by an electron beam that is injected into the cavity through an annular slot drilled on the left plate of the cavity. We see in Fig. 1 that the reflector has a twofold function: to prevent the radiation from leaking out of the system and to isolate electrically the gun (held at a negative potential) from the cavity (which is grounded). The reflector is specifically designed to have a center frequency around $6.6 \mathrm{GHz}$

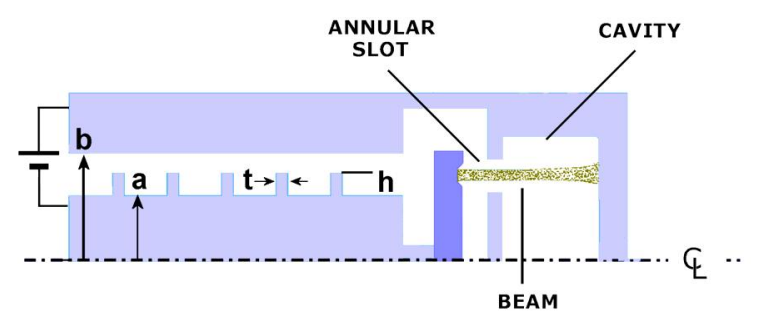

Figure 1. Monotron schematic with a Bragg reflector formed by a coaxial line capacitively loaded with circular disks

\section{Bragg reflector design and discussion}

As discussed in [5], which examines how stopband width, center frequency, and reflectivity are originated from the geometry and periodicity of the coaxial structure, we arrive at the design constraint $d>b-a$ to assure a stopband as widest as possible. The outer and inner radii of the coaxial line (Fig. 1) are selected as $b=3.53 \mathrm{~cm}$ and $a=$ $2.11 \mathrm{~cm}$ with finished dimensions close to those of commercially available metallic pipes. Following the above constraint, the periodic length is set at $d=1.80 \mathrm{~cm}$ and the disk thickness is chosen to be $t=0.35 \mathrm{~cm}(<<\mathrm{d})$ so as to avoid interception of the $\omega_{\pi^{+}}$and $\omega_{0}$-mode curves, as displayed in Fig. 2. Then we see that for $h=1.00 \mathrm{~cm}$ the monotron frequency of $6.6 \mathrm{GHz}$ lies midway in the 4.0-9.5 $\mathrm{GHz}$ stop band.

To verify the action of the reflector when coupled (through a $0.40-\mathrm{cm}$-width annular slot with average radius of $2.80 \mathrm{~cm}$ ) to a $\mathrm{TM}_{020}$-mode cavity, we consider first a 9.00 -cm-radius cavity operating at a frequency $(2.928$ $\mathrm{GHz}$ ) lying in the lower pass band. From a Superfish [6] simulation, Fig. 3(a) shows that, upon reflection from the open end at $z=15.45 \mathrm{~cm}$, it is formed along the periodic structure a standing-wave field with frequency of 2.953 $\mathrm{GHz}$, slightly above the closed-cavity frequency (2.928 $\mathrm{GHz})$, calculated by $f_{02}(\mathrm{GHz})=(15 / \pi) \mathrm{X}_{02} / \mathrm{R}_{\mathrm{w}}(\mathrm{cm})$, with $\mathrm{X}_{02}=5.520$. The scan at the ordinate $\mathrm{r}=2.8 \mathrm{~cm}$ shows in Fig. $3 b)$ that strong electric fields develop along the line.

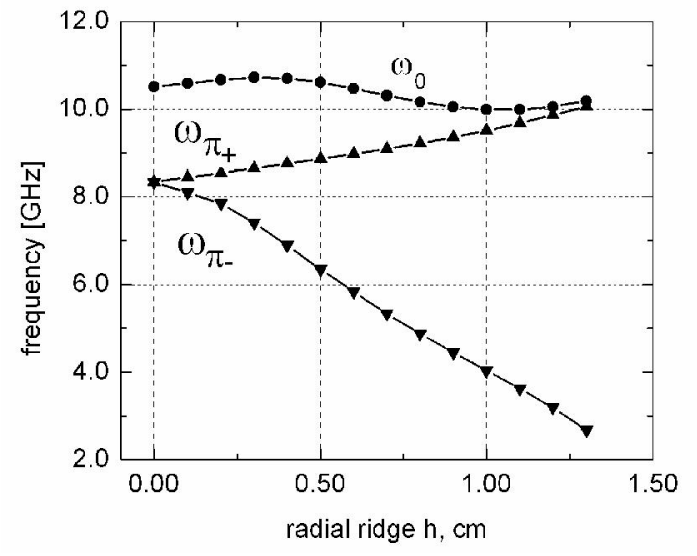

Figure 2. Calculated with Superfish [6], eigenfrequencies for zero and $\pi$ modes as a function of the height of the radial ridge, with corrugation parameters $\mathrm{b}=3.53 \mathrm{~cm}, \mathrm{a}=2.11 \mathrm{~cm}, \mathrm{~d}=1.80 \mathrm{~cm}$, and $\mathrm{t}=0.35 \mathrm{~cm}$ 


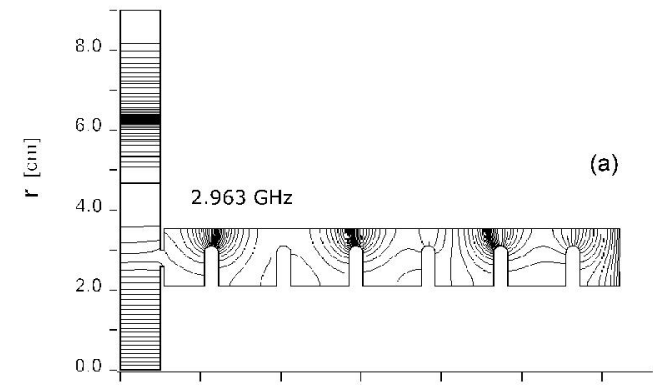

(b)

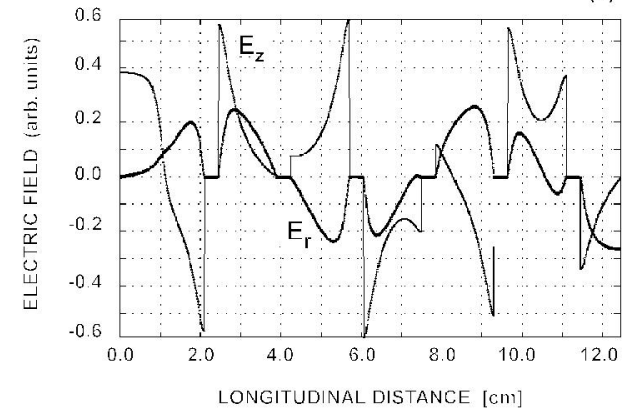

Figure 3. (a) Reflector coupled to a $\mathrm{TM}_{020}$-mode cavity of radius $R_{w}=9.0 \mathrm{~cm}$ with (b) longitudinal and radial electric field components along the ordinate $r=2.8 \mathrm{~cm}$

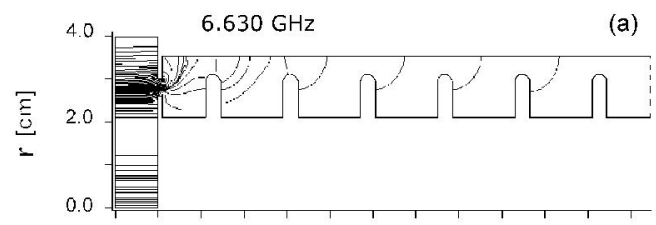

(b)

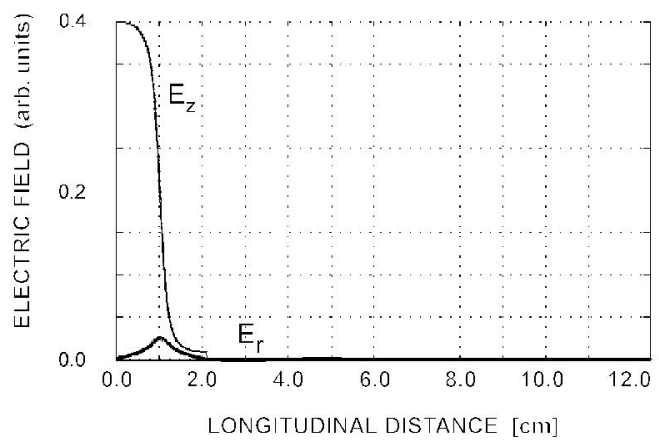

Figure 4. 4 The same as in Fig. 3, but with a cavity of radius $R_{w}=4.0 \mathrm{~cm}$

But when connecting the reflector to a $4.0-\mathrm{cm}$-radius cavity $\left(f_{02}=6.589 \mathrm{GHz}\right)$ we see in Fig. 4 that the leaking electric field (with Superfish-calculated frequency of 6.630 $\mathrm{GHz}$ ) is rapidly attenuated within a distance of $1.0 \mathrm{~cm}$. Similarly, with the electron gun intervening between the cavity and the corrugated line, as shown in Fig. 5, the fields are effectively suppressed past the first cell at $\mathrm{z}=6.0 \mathrm{~cm}$.

Thus, the efficacy of a coaxial Bragg reflector at preventing the RF fields from leaking out of a $6.6 \mathrm{GHz}$ monotron has been demonstrated with the inclusion of the electron gun between the cavity and the reflector. Having a tail extending up to the cathode surface, the effective RF electric-field axial distribution (Ez, in Fig. 17) is exactly like a transition profile, which, according to recent studies [17], is able to produce an electronic conversion efficiency as high as $\mathbf{5 0}$ percent.
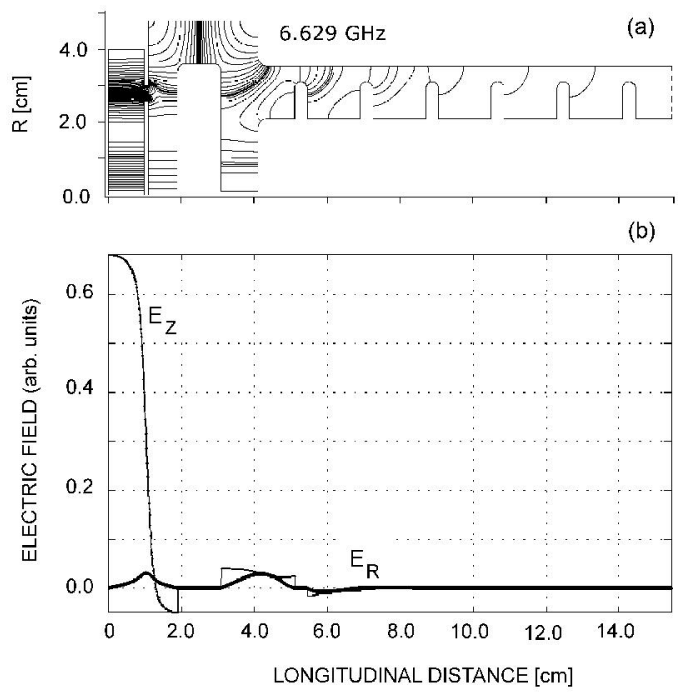

Figure 5. Coaxial Bragg reflector connected to the $6.6 \mathrm{GHz}$ monotron

\section{References}

1. T. Itoh and F-J. Hsu, IEEE Trans. Microwave Theory Tech., vol.27, no. 5, pp. 514-518, May 1979.

2. E. Yablonovitch, J. Opt. Soc. Am. B, vol. 10, no. 2, pp. 283295, February 1993.

3. V. L. Bratman, G. G. Denisov, N. S. Ginzburg, and M. I. Petelin, IEEE J. Quantum Electron., vol. 20, no. 3, pp. 282296, March 1983.

4. J. J. Barroso, IEEE Trans. Plasma Science, vol. 32, no.32, pp. 1205-121, June 2004.

5. J. J. Barroso and J. P. Leite Neto, Proc. Int. Microwave \& Optoelectron. Conf. (MMOC 2005), pp. 137-140, July 2005.

6. J. H. Billen and L. M. Young, "Poisson/Superfish", Rep. LA-UR-96-1934, Los Alamos National Lab., NM, 1996.

7. J. J. Barroso, IEEE Trans. Electron. Dev., vol. 52, no. 5, pp. 872-877, May 2005. 